\title{
Glucosylation of Isoflavonoids in Engineered Escherichia coli
}

\author{
Ramesh Prasad Pandey ${ }^{1,3}$, Prakash Parajuli ${ }^{1,3}$, Niranjan Koirala ${ }^{1}$, Joo Ho Lee ${ }^{1}$, Yong II Park ${ }^{2}$, and \\ Jae Kyung Sohng ${ }^{1, *}$
}

\begin{abstract}
A glycosyltransferase, YjiC, from Bacillus licheniformis has been used for the modification of the commercially available isoflavonoids genistein, daidzein, biochanin A and formononetin. The in vitro glycosylation reaction, using UDP- $\alpha$-D-glucose as a donor for the glucose moiety and aforementioned four acceptor molecules, showed the prominent glycosylation at $4^{\prime}$ and 7 hydroxyl groups, but not at the $5^{\text {th }}$ hydroxyl group of the A-ring, resulting in the production of genistein $4^{\prime}-O-\beta$-D-glucoside, genistein 7- $O$ $\beta$-D-glucoside (genistin), genistein $4,7-0-\beta$-D-diglucoside, biochanin A-7-O- $\beta$-D-glucoside (sissotrin), daidzein 4'-O- $\beta$ D-glucoside, daidzein 7- $O-\beta$-D-glucoside (daidzin), daidzein 4', 7-O- $\beta$-D-diglucoside, and formononetin 7-O- $\beta$-D-glucoside (ononin). The structures of all the products were elucidated using high performance liquid chromatographyphoto diode array and high resolution quadrupole time-offlight electrospray ionization mass spectrometry (HR QTOFESI/MS) analysis, and were compared with commercially available standard compounds. Significantly higher bioconversion rates of all four isoflavonoids was observed in both in vitro as well as in vivo bioconversion reactions. The in vivo fermentation of the isoflavonoids by applying engineered $E$. coli BL21(DE3)/ $\Delta$ pgi $\Delta z w f \Delta u s h A$ overexpressing phosphoglucomutase (pgm) and glucose 1-phosphate uridyltransferase (galU), along with YjiC, found more than $60 \%$ average conversion of $200 \mu \mathrm{M}$ of supplemented isoflavonoids, without any additional UDP- $\alpha$-D-glucose added in fermentation medium, which could be very beneficial to large scale industrial production of isoflavonoid glucosides.
\end{abstract}

\footnotetext{
'Department of Pharmaceutical Engineering, Institute of Biomolecule Reconstruction, Sun Moon University, Asan 336-708, Korea, ${ }^{2}$ Department of Biotechnology, The Catholic University of Korea, Bucheon 420743 , Korea, ${ }^{3}$ These authors contributed equally to this work.

*Correspondance: sohng@sunmoon.ac.kr
}

Received 21 November, 2013; revised 3 December, 2013; accepted 4 December, 2013; published online 19 February, 2014

Keywords: fermentation, glycosyltransferase, isoflavonoid glucosides, isoflavonoids

\section{INTRODUCTION}

Isoflavonoids are biologically active secondary metabolites which are produced by most leguminous plants. However, nonleguminous plants have also been reported to produce isoflavonoids. Structurally, isoflavonoids differ from flavonoids according to the position of the phenolic ring $\mathrm{B}$ attachment at the C-3 position of the C-ring, instead of at the C-2 position in flavonoids. A number of isoflavonoid derivatives (more than 1600 to date) have been identified from different sources (Veitch, $2007 ; 2013$ ). In plants, the biosynthetic pathway of most of the flavonoid groups of compound share the same chalcone intermediate (Pandey and Sohng, 2013). Isoflavonoids bifurcate from flavonoids after the formation of flavanone (either naringenin or liquiritigenin) by CYP450 enzyme isoflavone synthase (Lapcik, 2007; Pandey and Sohng, 2013). Isoflavones are further modified at different stages in plants to produce structurally diverse compounds, such as coumestan, coumaronochromone, rotenoid and pterocarpan. The genetics of isoflavonoids biosynthesis has been extensively studied in legume plants (Medicago truncatula, Glycine max) as well as other model plants (Arabidopsis thaliana, Beta vulgaris and Nicotiana tabacum (Veitch, 2013; Wang, 2010; 2011).

These low molecular weight phenolic compounds occur in free states as well as conjugated with sugar molecules. Most of those compounds are reported to exert several pharmacological properties, like estrogenic, antibacterial, antiviral, anti-inflammatory, endocrinological, antioxidant, anti-cancer properties (Di Carlo et al., 1999; Samuelsson and Bohlin, 2001). Soy beans are a major source of isoflavones such as daidzein and genistein (Dewick, 1993), thus soy is the most important source of isoflavone in human diet. Asian food contains high isoflavone content, as compared to western diets, and the daily intake reaches 20-50 mg per day, while being limited to $1 \mathrm{mg}$ per day in average western diets. Red clover (Trifolium pratens), a rich source of formononetin and biochanin A (4'-O-methlated forms of daidzein and genistein respectively), is used in organic farming as cow feed in Finland in order to generate isoflavones in organic cow milk (Hoikkala et al., 2007).

Flavonoids and isoflavonoids are diversified to large numbers of compounds by various post modifications. For example, methylation, prenylation, hydroxylation, acylation and glycosylation are common modifications of most of the compounds, and thus, bring about alterations in physical, chemical and biological

(c) The Korean Society for Molecular and Cellular Biology. All rights reserved.

(c) This is an open-access article distributed under the terms of the Creative Commons Attribution-NonCommercial-ShareAlike 3.0 Unported License. To view a copy of this license, visit http://creativecommons.org/licenses/by-nc-sa/3.0/. 
properties of the compounds (Botta et al., 2009; Kim et al., 2013; Wang, 2010). Glycosylation is one of the prominent modification reactions in plants, and is catalyzed by glycosyltransferases to transfer sugar moieties (glucose, galactose, rhamnose, xylose, rutinose etc.) to an acceptor molecule. The conjugation of a sugar moiety could be with reactive oxygen, carbon, nitrogen, or sulfur of the acceptor molecule. Glycosylation enhances the solubility and stability, and alters the biological properties of the compounds (Kren and Martinkova, 2001). The biological properties of most of the currently used therapeutics are derived from the sugar moiety attached to them (Weymouth-Wilson, 1997). Moreover, glycosylation could help in the detoxification of many compounds, and helps to keep the environment hygienic (Stupp et al., 2013; Vogt and Jones, 2000).

In this study, we applied Bacillus licheniformis DSM-13 glycosyltransferase (YjiC) to modify four commercially available pharmacologically potent isoflavonoids. YjiC has been determined to catalyze glycosylation of chalcone (phloretin) (Pandey et al., 2013a), flavonols (Pandey et al., 2013b), flavone (apigenin) (Gurung et al., 2013) and geldanamycin analogues (Wu et al., 2012). Moreover, it has been found that YjiC is flexible to glycosylate at maximum available reactive hydroxyl groups to produce diverse kinds of glucoside derivatives. Hence, here we attempt the modification of different isoflavonoids, i.e., biochanin $A$, daidzein, formononetin and genistein, to produce their glucoside derivatives using in vitro as well as in vivo approaches.

\section{MATERIALS AND METHODS}

\section{General procedures}

Genistein, daidzein, biochanin A and formononetin were purchased from Tokyo Chemical Industry (Japan). UDP- $\alpha$-D-glucose was purchased from Sigma-Aldrich (USA). Genistein 7-O$\beta$-D-glucoside (genistin) was available in our laboratory. All other chemicals and reagents were of the highest chemical grade available. The high resolution mass spectrometry spectra were obtained in positive ion mode on ACQUITY (UPLC, Waters Corp., USA) coupled with SYNAPT G2-S (Waters Corp.). The details of the methodology for the cloning, expression, and purification of YjiC are described in our previous report (Pandey et al., 2013a).

\section{General lab-scale glycosyltransferase enzymatic reaction} The YjiC reactions were carried out in a total reaction mixture volume of $200 \mu \mathrm{l}$, containing $100 \mathrm{mM}$ Tris-Cl (pH 8.0), $4 \mathrm{mM}$ UDP- $\alpha$-D-glucose, $2 \mathrm{mM}$ acceptor substrates (genistein, daidzein, biochanin A and formononetin), $10 \mathrm{mM} \mathrm{MgCl}_{2}$, and $50 \mu \mathrm{g}$ of appropriately diluted enzyme. The reaction mixtures were incubated at $37^{\circ} \mathrm{C}$ for $3 \mathrm{~h}$. Assay mixtures lacking enzyme served as controls. The reaction was quenched by adding 400 $\mu \mathrm{l}$ chilled methanol and mixed by vortexing for several minutes. Then, the aliquots were centrifuged at $12,000 \mathrm{rpm}$ to remove proteins, and the reaction product was directly monitored by High Pressure Liquid Chromatography-Photo Diode Array (HPLC-PDA) and HR QTOF-ESI/MS after proper dilution.

\section{Bioconversion using engineered $E$. coli}

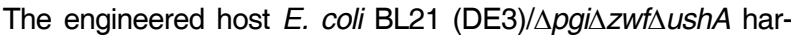
boring recombinant plasmids (pETDuet-nfa44530-galU and pET28-yjiC) was used for the whole cell biocatalyst assay. 200 $\mu$ of overnight culture of the above strain was transferred to 50 $\mathrm{ml}$ of TB liquid medium containing antibiotics [kanamycin (50 $\mu \mathrm{g} / \mathrm{ml})$ and ampicillin $(100 \mu \mathrm{g} / \mathrm{ml})]$, which was further supple- mented with an additional $1 \%$ mannitol, $1 \%$ glucose, $1 \%$ fructose and $1 \%$ glycerol. The culture was incubated at $37^{\circ} \mathrm{C}$ until the optical density at $600 \mathrm{~nm}\left(\mathrm{OD}_{600 \mathrm{~nm}}\right)$ reached approximately 0.6 . Then, $0.5 \mathrm{mM}$ of IPTG was added to the final concentration, and the cultures were incubated at $20^{\circ} \mathrm{C}$ for $16 \mathrm{~h}$. To the above culture, $0.2 \mathrm{mM}$ of each isoflavonoid (genistein, daidzein, formononetin and biochanin A) was added, and incubation was continued at $20^{\circ} \mathrm{C}$ for $48 \mathrm{~h}$. $500 \mu \mathrm{l}$ of samples were taken at 48 $\mathrm{h}$, centrifuged, and extracted by a double volume of ethyl acetate. The organic layer was dried and was again dissolved in $500 \mu \mathrm{l}$ of HPLC grade methanol for HPLC-PDA analysis. The conversion percentage was determined by HPLC and was then calculated by dividing the integrated area of glucosylated products by the sum of the integrated area of the products, plus the integrated area of the remaining acceptor substrate.

\section{Analytical method}

The reverse-phase HPLC-PDA analysis was performed with a $\mathrm{C}_{18}$ column (Mightysil RP-18 GP $(4.6 \times 250 \mathrm{~mm}, 5 \mu \mathrm{m})$ connected to a PDA $\left(26 \mathrm{~nm}_{\mathrm{nm}}\right)$ using binary conditions of $\mathrm{H}_{2} \mathrm{O}(0.1 \%$ trifluroacetic acid buffer) and $100 \%$ acetonitrile $(\mathrm{ACN})$ at a flow rate of $1 \mathrm{ml} / \mathrm{min}$ for $25 \mathrm{~min}$. The ACN concentrations were as follows: $20 \%$ (0-5 min), $50 \%$ (5-10 $\mathrm{min}$ ), $70 \%$ (10-15 $\mathrm{min}$ ), $90 \%$ (15-20 $\mathrm{min}), 10 \%$ (20-25 $\mathrm{min})$.

\section{RESULTS}

Recombinant protein production and in vitro glucosylation reactions

pET28a-YjiC constructed was transformed in E. coli BL21(DE3) and used for the production of approximately $46 \mathrm{kDa}$ hexahistagged YjiC protein (Pandey et al., 2013a; 2013b). The protein was purified using $\mathrm{Ni}^{2+}$ chelate affinity chromatography and was concentrated using a $30 \mathrm{~K}$ cut-off Amicon ultra centrifugale filter. The concentrated purified protein was quantified and used for the in vitro glycosyltransferase reactions with four different commercially available isoflavonoids. The in vitro reaction was carried out as described in "Material and Methods" for individual isoflavonoids. After the incubation of reaction mixture at $37^{\circ} \mathrm{C}$ for $3 \mathrm{~h}$, it was quenched with chilled methanol and analyzed by HPLC-PDA analysis.

\section{Liquid chromatography analysis}

The HPLC-PDA analysis of all four individual reaction mixtures was carried out under identical HPLC conditions. The genistein reaction mixture showed three glucosylated products- $\mathrm{G}_{1}, \mathrm{G}_{2}$ and $\mathrm{G}_{3}$ at retention times $\left(t_{R}\right)$ of $14.2 \mathrm{~min}, 13.7 \mathrm{~min}, 11.8 \mathrm{~min}$ respectively. Similarly, three glucosylated products $\left(D_{1}, D_{2}\right.$, and $\mathrm{D}_{3}$ with $t_{R} 13.4 \mathrm{~min}, 13.2 \mathrm{~min}$ and $11.4 \mathrm{~min}$ respectively) were observed with daidzein. However, a single product was found to be produced in the case of biochanin $A$ and formononetin (Fig. 2). Genistein has three hydroxyl groups at 4', 5 and 7 positions (Fig. 1). Since, the previous studies showed the flexible activity of $\mathrm{YjiC}$, we could not predict the exact position of glucosylation in compounds having multiple hydroxyl groups. Thus, we compared the $t_{R}$ of genistin (genistein-7-O- $\beta$-D-glucoside) to identify the products of genistein (Fig. 2A). The $t_{R}$ of genistein was found to be exactly with the same as that of $G_{2}$, confirming one of the products of genistein to be genistein-7-O$\beta$-D-glucoside. Though daidzein has only two prominent reactive hydroxyl groups, at 4' and 7 positions (Fig. 1), three glucosylated products were observed. In contrast, biochanin A also has two reactive hydroxyl positions at 5 and 7 carbons of A-ring, but has only one glucosylated product. This results shows that 
<smiles>COc1cccc2c(=O)c(-c3ccccc3)coc12</smiles><smiles>O=c1c(-c2ccc(O)cc2)coc2cc(O)ccc12</smiles>

Genistein

Daidzein<smiles>COc1ccc(-c2coc3cc(O)ccc3c2=O)cc1</smiles>

Biochanin A

Formononetin

Fig. 1. A typical structure of isoflavonoid along with four different commercially available isoflavonoids (genistein, biochanin A, daidzein and formononetin) used in this study.

YjiC could not be able to glucosylate at $5^{\text {th }}$ hydroxyl position of A-ring in isoflavonoids. A similar observation was also previously noticed when YjiC was used for the reaction of apigenin (Gurung et al., 2013). The NMR analysis of all the reaction products found that there was no glycosylation at the $5^{\text {th }}$ hydroxyl position of the A-ring. However, further analysis of all the reaction products by high resolution mass spectrometry was carried out to identify the possible structures of the compounds.

\section{Structural elucidation by high resolution mass spectrometry analysis}

The HPLC-PDA coupled with HR QTOF-ESI/MS analysis of the all reaction products was carried out for the identification of the products (Fig. 3). The exact mass analysis of genistein reaction mixture showed two monoglucosides $\left(\mathrm{G}_{1},[\mathrm{M}+\mathrm{H}]^{+} \mathrm{m} / \mathrm{z}^{+}\right.$ $\sim 433.1131$ and $G_{2},[M+H]^{+} m / z^{+} \sim 433.1140$ ) whereas $G_{3}$ was determined as a diglucoside $\left([\mathrm{M}+\mathrm{H}]^{+} \mathrm{m} / \mathrm{z}^{+} \sim 595.1649\right)$ of genistein. This result confirmed $\mathrm{G}_{1}$ as genistein $4^{\prime}-O-\beta$-D-glucoside, $\mathrm{G}_{2}$ as genistein 7-O- $\beta$-D-glucoside and $\mathrm{G}_{3}$ as genistein $4^{\prime}, 7-O-$ $\beta$-D-diglucoside. Since, there was no exact mass matching with the triglucoside of genistein, we confirmed that YjiC could not be able to glucosylate at the 5-hydroxyl position of the A-ring of isoflavonoids. Further evidence of the production of a monoglucoside of biochanin A, biochanin A-7-O- $\beta$-D-glucoside $\left([\mathrm{M}+\mathrm{H}]^{+}\right.$ $\left.\mathrm{m} / \mathrm{z}^{+} \sim 447.1350\right)$ supported our result. This could be because of the hindrance of the ketone group present in the C-ring of flavonoids and isoflavonoids. In the case of daidzein, we also found two monoglucosides $\left(D_{1},[M+H]^{+} \mathrm{m} / \mathrm{z}^{+} \sim 417.1203\right.$ and $D_{2}$, $\left.[\mathrm{M}+\mathrm{H}]^{+} \mathrm{m} / \mathrm{z}^{+} \sim 4417.1178\right)$ and a diglucoside $\left(\mathrm{D}_{3},[\mathrm{M}+\mathrm{H}]^{+} \mathrm{m} / \mathrm{z}^{+} \sim\right.$ 579.1711). The 4 '-hydroxyl position glucosylated product has been found to have a longer retention time than the 7-hydroxyl position glucosylated product in the case of apigenin (gurung et
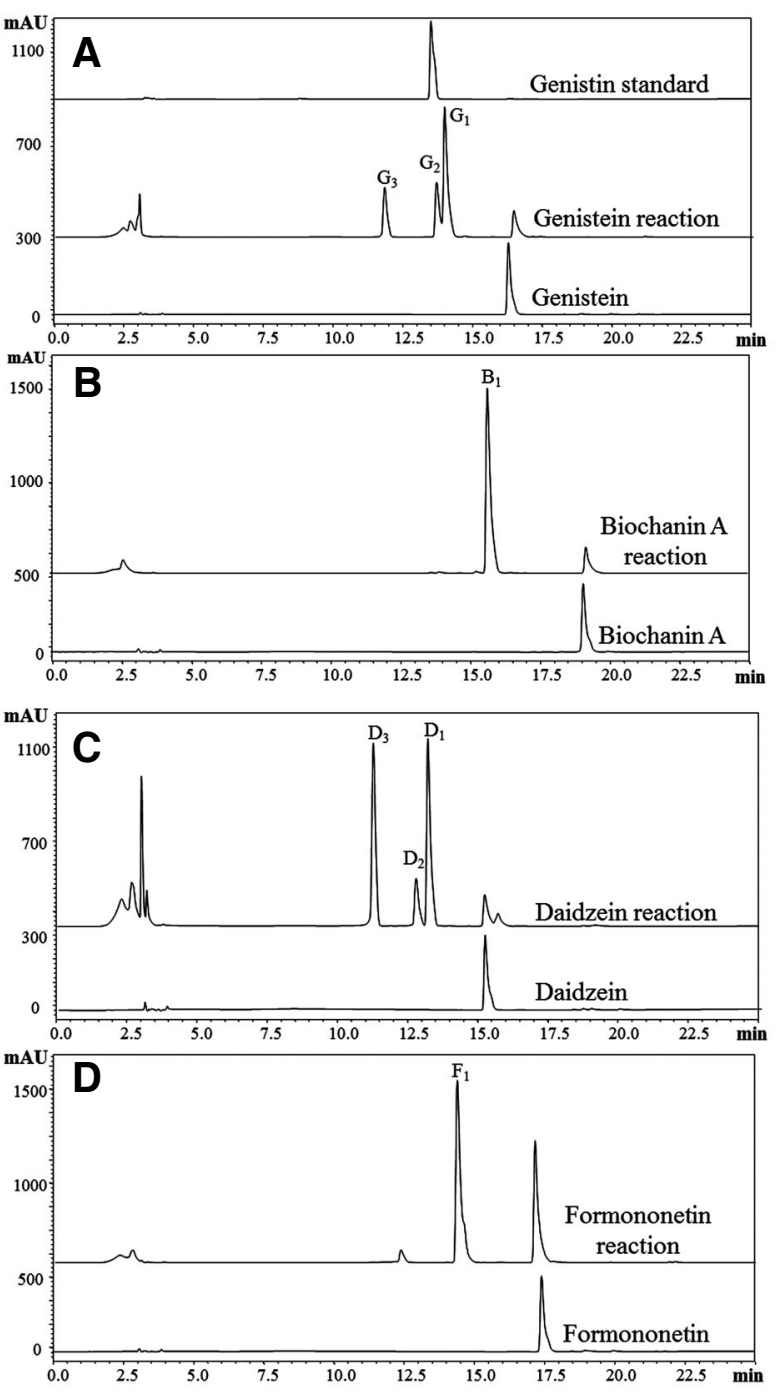

Fig. 2. HPLC chromatogram of reaction products of four different isoflavonoids. (A) genistein, $(B)$ biochanin $A,(C)$ daidzein and (D) formononetin. The Figure $A$ contains an additional chromatogram of standard genistin (genistein 7-O- $\beta$-D-glucoside) at the top.

al., 2013) and genistein too. Thus, we confirmed $D_{1}$ as daidzein 4'-O- $\beta$-D-glucoside and $\mathrm{D}_{2}$ as daidzein 7-O- $\beta$-D-glucoside. Since there are only two possible reactive hydroxyl groups in daidzein, the diglucoside derivative $\left(D_{3}\right)$ has been confirmed as daidzein 4 ', 7-O- $\beta$-D-diglucoside. The single product of formononetin was also identified as formononetin 7-O- $\beta$-D-glucoside $\left([\mathrm{M}+\mathrm{H}]^{+} \mathrm{m} / \mathrm{z}^{+} \sim 431.1355\right)$ as it has only one available hydroxyl group at the 7 position for glucosylation.

\section{Study of conversion rate of compounds}

We studied the conversion percentage of each isoflavonoid by HPLC analysis. The comparative conversion rate analysis showed maximum conversion of daidzein to beapproximately $92 \%$. However, the conversion rate of genistein, biochanin $A$ and formononetin was $69 \%, 74.4 \%$ and $71.5 \%$, respectively (Fig. 4). The previous studies in our laboratory showed relatively higher conversion percentages of flavonols, such as $98 \%$ conversion 
A

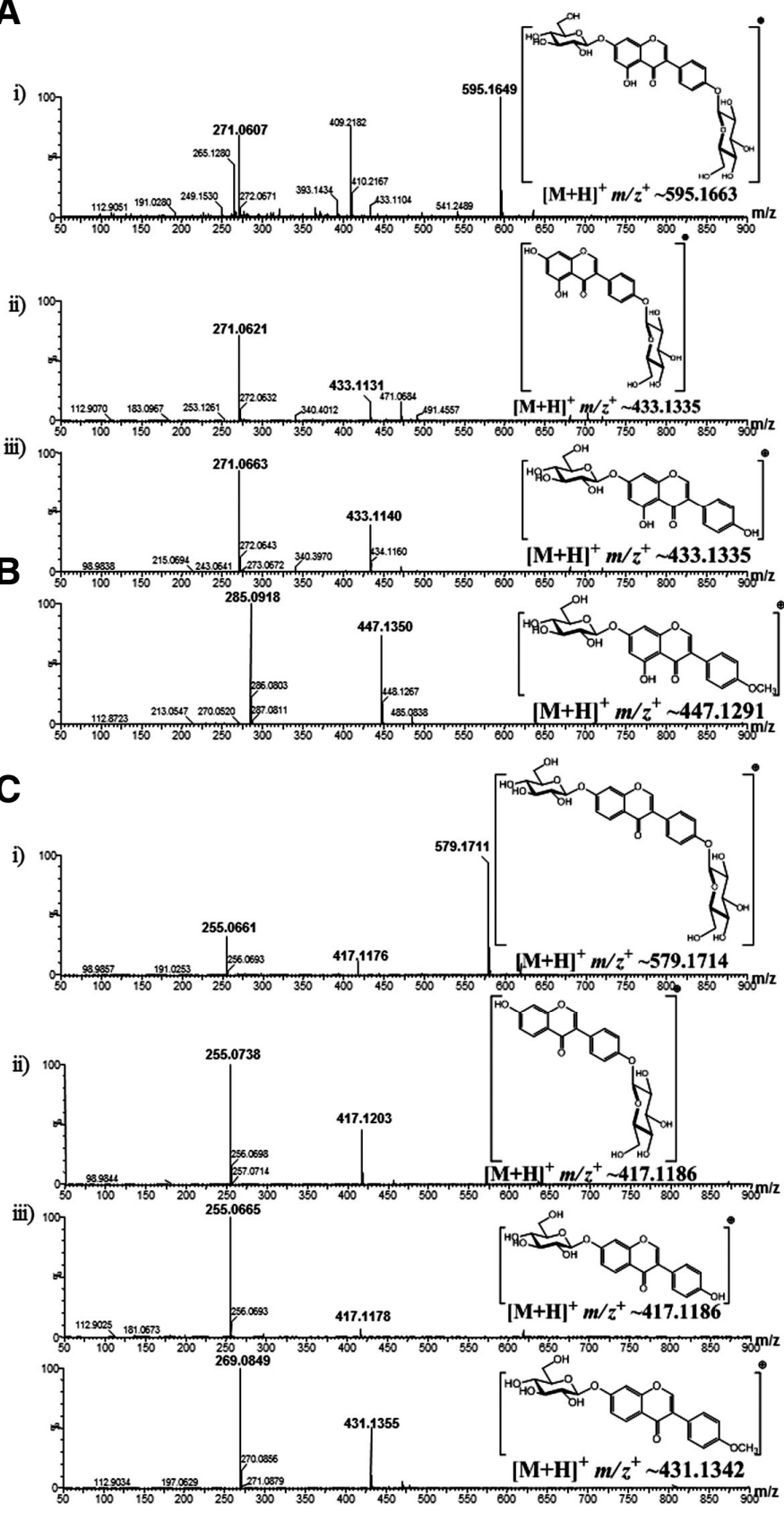

Fig. 3. HPLC-PDA-QTOF-HR ESI/MS analysis. (A) Glucosides of genistein: (i) genistein $4,7-O-\beta$-D-diglucoside $\left(G_{3}\right.$, a diglucoside of genistein), (ii) genistein $4^{\prime}-O-\beta$-Dglucoside $\left(\mathrm{G}_{1}\right)$ and (iii) genistein 7-O- $\beta$-D-glucoside $\left(\mathrm{G}_{2}\right)$ (two different monoglucosides of genistein), (B) biochanin A 7- $O$ - $\beta$-D-glucoside ( $\mathrm{B}_{1}$, a monoglucoside of biochanin $\mathrm{A}$ ), (C) Glucosides of daidzein: (i) daidzein 4',7-O- $\beta$-Ddigluco-side $\left(\mathrm{D}_{3}\right.$, a diglucoside of daidzein), (ii) daidzein $4^{\prime}$ $O$ - $\beta$-D-glucoside $\left(\mathrm{D}_{1}\right)$ and (iii) daidzein 7-O- $\beta$-D-glucoside $\left(D_{2}\right)$ (two different monoglucosides of daidzein), and (D) formononetin $7-O-\beta$-D-glucoside $\left(F_{1}\right.$, a monoglucoside of formononetin).

of fisetin, approximately $100 \%$ conversion of kaempferol (Pandey et al., 2013b), and with phloretin (chalcone) the conversion was 95\% (Pandey et al., 2013a). Based on those previous results, the catalysis activity of $\mathrm{YjiC}$ is lower with isoflavonoids. However, the conversion was significantly higher with daidzein. With this significant in vitro bioconversion result, we applied the YjiC glycosyltransferase for in vivo bioconversion of those isoflavonoids by engineering $E$. coli BL21 (DE3).

Bioconversion of isoflavonoids using engineered $E$. coli As described in our previous reports, we used optimized medium for the engineered $E$. coli strain $E$. coli BL21 (DE3)/ $\Delta p g i$ $\Delta z w f \Delta u s h A$ over-expressing phosphoglucomutase (nfa44530 from Nocardia farcinica) and glucose 1-phosphate uridyl transferase (galU from E. coll) (Fig. 5A), based on their cell growth (Malla et al., 2013; Pandey et al., 2013c) and determined the biotransformation of all isoflavonoids. The biotransformation resulted in good conversion of all isoflavonoids to their glucosides, showing biochanin $\mathrm{A}$ with the highest conversion rate $(\sim 75 \%)$ and formononetin has $\sim 45 \%$ conversion being the lowest conversion rate among all isoflavonoids used in this study (Fig. 5B). Though daidzein had the highest conversion rate in the in vitro reaction (Fig. 4), the in vivo bioconversion was found to be lowered to $62 \%$. However, this approach could be used to scale up fermentation in large scale fermentors, to produce large quantities of target compounds for commercial purposes, 


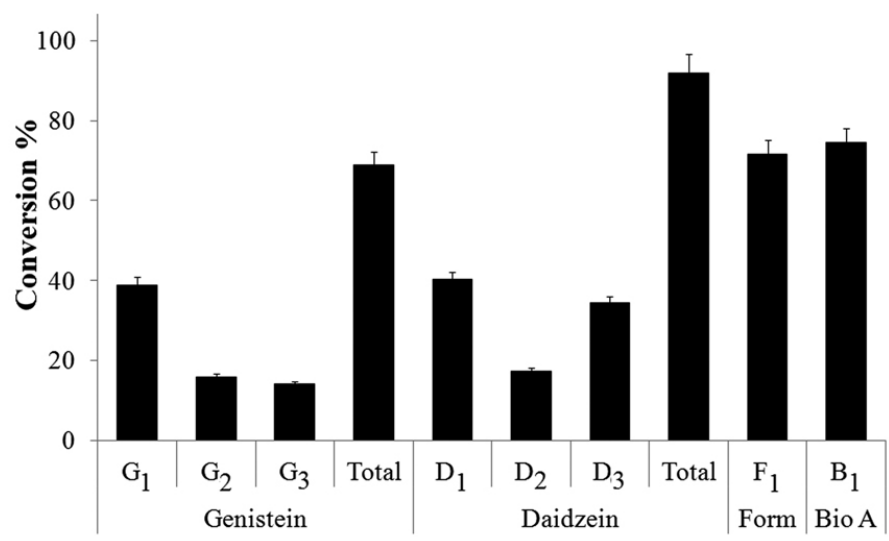

Substrates
Fig. 4. Conversion rate of each isoflavonoids [genistein, daidzein, formononetin (Form), and biochanin A (Bio A)] and formation rate of each glucosylated products catalyzed by YjiC under identical conditions. Total indicates the sum of formation rate of each monoglucosides $\left(G_{1}, G_{2}\right)$ and diglucoside $\left(G_{3}\right)$ of genistein and monoglucosides $\left(D_{1}\right.$, $D_{2}$ ) and diglucoside $\left(D_{3}\right)$ of daidzein respectively. $B_{1}$ and $\mathrm{F}_{1}$ are the 7-O- $\beta$-D-glucoside of biochanin $\mathrm{A}$ and formononetin respectively. The error bars show the standard deviations of three independent experiments, which were less than $5 \%$.
A

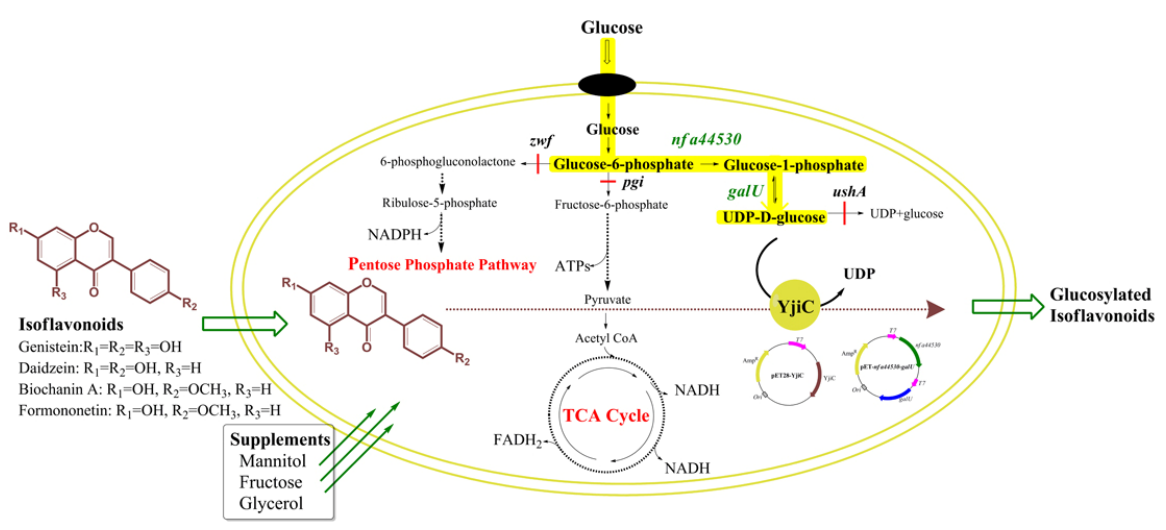

B

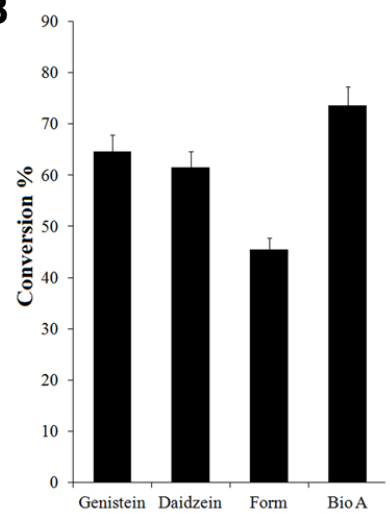

Fig. 5. Bioconversion of isoflavonoids using engineered E. coli BL21 (DE3). (A) Diagramatic sketch of engineered E. coli. Pool of UDP- $\alpha$-Dglucose has been expected to be increased by over expressing phosphoglucomutase (nfa44530) and uridyltransferase (gall) and engineering $E$. coli $\mathrm{BL21}(\mathrm{DE} 3)$ by knock-out of glucose phosphate isomerase (pgl), D-glucose-6-phosphate dehydrogenase (zwf) and UDP- $\alpha$-D-glucose hydrolase (ushA) genes. The yellow highlighted pathway was over expressed. (B) The conversion percentage of each isoflavonoids in shake flask cultures of engineered E. coli BL21(DE3)/ $\Delta$ pgi zwf $\Delta$ ushA pET-Duet-nfa44530-galU, pET28- yjiC supplemented with $0.2 \mathrm{mM}$ of each isoflavonoids in independent experiments. The ethyl acetate extract of the $48 \mathrm{~h}$ culture incubated at $20^{\circ} \mathrm{C}$ by HPLC-PDA. The conversion percentage was determined by HPLC and calculated by dividing the integrated area of glucosylated products by the sum of the integrated area of the products plus the integrated area of the remaining acceptor substrate. Form represents formononetin whereas BioA is biochanin A. The error bars show the standard deviations of three independent experiments, which were less than $5 \%$.

which could lead to the availability of the compound at a lower price. The further optimization of production and fermentation processes, as well as the engineering of $\mathrm{YjiC}$, is essential for the attainment of higher production levels, as well as for the regiospecific production of target compound.

\section{DISCUSSION}

Engineering microbial cells and applying them as a microbial cell factories for the production of valuable products has attracted a wide range of industries for the eco-friendly production of medicinal compounds, cosmetics, and other commodities. Thus, we have applied engineered $E$. coli for the efficient and cheap bioconversion of isoflavonoids to their glucoside derivatives. The in vitro biotransformation of compounds to their glycosides for large scale production requires expensive nucleotide sugar donors (for example, UDP- $\alpha$-D-glucose) and purified glycosyltransferase enzymes. Hence, the process is expensive, and cannot be readily scaled up. Similarly, the chemical approach of production of those glycosides requires multiple steps of chemical reactions, which are time consuming and tedious, and the entire process is not eco-friendly. Hence, the green biotechnology approach of using microbial cell factories has a number of merits over in vitro and chemical approaches of conversion of compounds to their glycoside derivatives by simple fermentation (Williams et al., 2011).

Metabolically engineered microbes (E. coli, Saccharomyces cerevisiae, and Streptomyces species) have been widely used to diversify natural products. Artificial biosynthetic pathways are introduced in such microbes for the production of plant specific medicinal polyketides, including isoflavonoids, flavonoids, stilbenoids etc (Horinouchi, 2008). By using this biosynthesis approach, natural as well as unnatural compounds have been developed, which could have novel properties (Hopwood et al., 
1985; Julsing et al., 2006; McDaniel et al., 1999; Ververidis et al., 2007). In fact, a number of studies have been carried out and a huge number of compounds have been generated, with a library of those compounds having been constructed. Since flavonoids and isoflavonoids have been identified as potentially having beneficial effects on human health, metabolic engineering has been carried out to modify them, thus increasing the yield of the target compounds.

We developed the E. coli BL21(DE3) expression host as a microbial cell factory by over-expressing UDP- $\alpha$-D-glucose biosynthetic pathway genes and directing the carbon flow towards UDP- $\alpha$-D-glucose by blocking the genes diverting the precursors of the UDP- $\alpha$-D-glucose. Glucose phosphate isomerase (pgi), D-glucose-6-phosphate dehydrogenase (zwf) and UDP$\alpha$-D-glucose hydrolase (ushA) genes were blocked by deleting their respective genes from the chromosome of $E$. coli (Pandey et al., 2013c). The blocking of pgi and $z w f$ diverts the flow of glucose 6-phosphate by glycolysis and pentose phosphate pathways, resulting in the increased pool of UDP- $\alpha$-D-glucose in cell cytoplasm, which is utilized by glycosyltransferase to produce glucosides of the target compounds. UshA hydrolyzes the excess UDP- $\alpha$-D-glucose to UDP and glucose. Thus, the $E$. coli BL21(DE3) strain lacking pgi, zwf and ushA genes and over-expressing nfa44530 and galU (Fig. 5A) could serve as a suitable strain for glycosylation. Since, E. coli BL21 (DE3)/Apgi $\triangle z w f \Delta u s h A$ has shown better performance in bioconversion of quercetin to quercetin 3-O-xyloside (Pandey et al., 2013c), in which UDP- $\alpha$-D-glucose is an intermediate of UDP-xylose biosynthetic pathway, than E. coli BL21 (DE3), E. coli BL21 (DE3)/

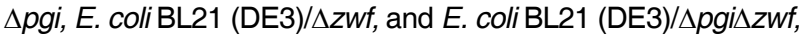
we have selected $E$. coli BL21 (DE3)/ $\Delta p g i \Delta z w f \Delta u s h A$ for the bioconversion of isoflavonoids to their respective glucosides.

Importantly, the glucosides of isoflavonoids like genistein 7$O-\beta$-D-glucoside (Genistin), daidzein7- $O-\beta$-D-glucoside (Daidzin), formononetin 7-O- $\beta$-D-glucoside (Ononin), biochanin A 70 - $\beta$-D-glucoside (Sissotrin) have relatively very high prices. Few of the monoglucosides and diglucosides are commercially unavailable. In this context, the production of these isoflavonoid glucoside derivatives and their proper commercialization could be an exciting prospect.

\section{ACKNOWLEDGMENTS}

This study was supported by the Converging Research Center Program (2012K001387), the Ministry of Science, ICT and Future Planning and by the grant from the Next-Generation BioGreen 21 Program (JKS: PJ0094832), Rural Development Administration, and Republic of Korea.

\section{REFERENCES}

Botta, B., Menendez, P., Zappia, G., de Lima, R.A., Torge, R., and Monachea, G.D. (2009). Prenylated Isoflavonoids: Botanical distribution, structures, biological activities and biotechnological studies. An update (1995-2006). Curr. Med. Chem. 16, 3414-3468.

Dewick, P.M. (1993). The flavonoids, advances in research since 1986. In Isoflavonoids, J.B. Harbone, ed. (London: Chapman and Hall), pp. 117-238.

Di Carlo, G., Mascolo, N., Izzo, A.A., and Capasso, F. (1999). Flavonoids: old and new aspects of a class of natural therapeutic drugs. Life Sci. 65, 337-353.

Gurung, R.B., Kim, E.H., Oh, T.J., and Sohng, J.K. (2013). Enzymatic synthesis of apigenin glucosides by glucosyltransferase (YjiC) from Bacillus licheniformis DSM 13. Mol. Cells 36, 1-10.

Hoikkala, A., Mustonen, E., Saastamoinen, I., Jokela, T., Taponen, J., Saloniemi, H., and Wähälä, K. (2007). High levels of equol in organic skimmed Finnish cow milk. Mol. Nutr. Food Res. 51, 782-786.
Hopwood, D.A., Malpartida, F., Kieser, H.M., Ikeda, H., Duncan, J., Fujii, I., Rudd, B.A.M., Floss, H.G., and Omura, S. (1985). Production of "hybrid" antibiotics by genetic engineering. Nature 314 642-644

Horinouchi, S. (2008). Combinatorial biosynthesis of non-bacterial and unnatural flavonoids, stilbenoids and curcuminoids by microorganisms. J. Antibiot. (Tokyo) 61, 709-728.

Julsing, M.K., Koulman, A., Woerdenbag, H.J., Quax, W.J., and Kayser, O. (2006). Combinatorial biosynthesis of medicinal plant secondary metabolites. Biomol. Eng. 23, 265-279.

Kren, V., and Martinkova, L. (2001). Glycosides in medicine: "The role of glycosidic residue in biological activity". Curr. Med. Chem. 8, 1303-1328.

Kim, B., Choi, J.S., Yi, E.H., Lee, J., Won, C., Ye, S., and Kim, M. (2013). Relative antioxidant activities of quercetin and its structurally related substances and their effects on NF-KB/CRE/AP-1 signalling in murine macrophages. Mol. Cells 35, 410-420.

Lapcik, O. (2007). Isoflavonoids in non-leguminous tax: a rarity or a rule? Phytochemistry 68, 2909-2916.

Malla, S., Pandey, R.P., Kim, B.G., and Sohng, J.K. (2013). Regiospecific modifications of naringenin for astragalin production in Escherichia coli. Biotechnol. Bioeng. 110, 2525-2535.

McDaniel, R., Thamchaipenet, A., Gustafsson, C., Fu, H., Betlach, M., and Ashley, G. (1999). Multiple genetic modifications of the erythromycin polyketide synthase to produce a library of nove "unnatural" natural products. Proc. Natl. Acad. Sci. USA 96, 1846-1851.

Pandey, R.P., and Sohng, J.K. (2013). Genetics of flavonoids. In Hand Book of Natural Products, Phytochemistry, Botany and Metabolism, K.G. Ramawat, and J.M. Mérillon, eds. (Berlin Heidelberg: Springer-Verlag), pp.1617-1645.

Pandey, R.P., Li, T.F., Kim, E.H., Yamaguchi, T., Park, Y.I., Kim, J.S., and Sohng, J.K. (2013a). Enzymatic synthesis of novel phloretin glucosides. Appl. Environ. Microbiol. 79, 3516-3521.

Pandey, R.P., Parajuli, P., Koirala, N., Park, J.W., and Sohng, J.K (2013b). Probing 3-hydroxyflavone for in vitro glycorandomization of flavonols by YjiC. Appl. Environ. Microbiol. 79, 6833-6838

Pandey, R.P., Malla, S., Simkhada, D., Kim, B.G., and Sohng, J.K. (2013c). Production of 3-O-xylosyl quercetin in Escherichia coli. Appl. Microbiol. Biotechnol. 97, 1889-1901.

Samuelsson, G., and Bohlin, L. (2001). Drugs of Natural Origin: A textbook of pharmacognosy. (Stockholm: Swedish Pharmaceutical Press).

Stupp, G.S., von Reuss, S.H., Izrayelit, Y., Ajredini, R., Schroeder, F.C., and Edison, A.S. (2013). Chemical detoxification of small molecules by Caenorhabditis elegans. ACS Chem. Biol. 15, 309-313

Veitch, N.C. (2007). Isoflavonoids of the Leguminosae. Nat. Prod. Rep. 24, 417-464.

Veitch, N.C. (2013). Isoflavonoids of the Leguminosae. Nat. Prod. Rep. 30, 988-1027.

Ververidis, F., Trantas, E., Douglas, C., Vollmer, G., Kretzschmar, G., and Panopoulos, N. (2007). Biotechnology of flavonoids and other phenylpropanoid-derived natural products. Part II: Reconstitution of multienzyme pathways in plants and microbes. Biotechnol. J. 2, 1235-1249.

Vogt, T., and Jones, P. (2000). Glycosyltransferases in plant natural product synthesis: characterization of a supergene family. Trends Plant Sci. 5, 380-386.

Wang, X. (2010). Structural studies and mechanisms of isoflavonoid biosynthesis. In Isoflavones Biosynthesis, Occurance and Health Effects. M.J. Thompson, ed. (New York: Nova Science Publishers Inc.), pp. 239-254.

Wang, X. (2011). Structure, function, and engineering of enzymes in isoflavonoid biosynthesis. Funct. Integr. Genomics 11, 13-22.

Weymouth-Wilson, A.C. (1997). The role of carbohydrates in biologically active natural products. Nat. Prod. Rep. 14, 99-110.

Williams, G.J., Yang, J., Zhang, C., and Thorson, J.S. (2011). Recombinant $E$. coli prototype strains for in vivo glycorandomization. ACS Chem. Biol. 6, 95-100.

Wu, C.Z., Jang, J.H., Woo, M., Ahn, J.S., Kim, J.S., and Hong, Y.S. (2012) Enzymatic glycosylation of non-benzoquinone geldanamycin analogs via Bacillus UDP-glycosyltransferase. Appl. Environ. Microbiol. 78, 7680-7686. 